Revue québécoise de psychologie

Wuevécécolise

\title{
INTERVENIR COLLECTIVEMENT SUR LE TRAVAIL QUI FAIT SOUFFRIR : ENTRE SOURCES DE TENSION ET DÉVELOPPEMENT DU POUVOIR D’AGIR D’UN COMITÉ SANTÉ À L'ÉCOLE INTERVENING COLLECTIVELY ON WORK RELATED SUFFERING: BETWEEN SOURCES OF TENSION AND POWER TO ACT OF A SCHOOL HEALTH COMITY
}

\section{Emmanuel Poirel et Marc-André Houde}

Volume 40, numéro 3, 2019

LA SANTÉ MENTALE À L'ÉCOLE : ENJEUX ET DÉFIS DE LA PROMOTION ET L'ACCOMPAGNEMENT

MENTAL HEALTH AT SCHOOL: CHALLENGES AND CHALLENGES OF PROMOTION AND SUPPORT

URI : https://id.erudit.org/iderudit/1067554ar

DOI : https://doi.org/10.7202/1067554ar

Aller au sommaire du numéro

Éditeur(s)

Revue québécoise de psychologie

ISSN

2560-6530 (numérique)

Découvrir la revue

Citer cet article

Poirel, E. \& Houde, M.-A. (2019). INTERVENIR COLLECTIVEMENT SUR LE TRAVAIL QUI FAIT SOUFFRIR : ENTRE SOURCES DE TENSION ET DÉVELOPPEMENT DU POUVOIR D’AGIR D’UN COMITÉ SANTÉ À L'ÉCOLE. Revue québécoise de psychologie, 40(3), 169-185.

https://doi.org/10.7202/1067554ar

\section{Résumé de l'article}

Les risques pour la santé mentale en milieu scolaire au Québec sont nombreux et les salariés du secteur de l'enseignement seraient parmi les plus atteints par la détresse psychologique. Considérant que les interventions préventives individuelles sont insuffisantes et peinent à enrayer ce mal, nous présentons les fruits d'une recherche-intervention inspirée de la clinique de l'activité qui montre que la santé peut se construire par le pouvoir d'agir dans un contexte offrant la possibilité de générer de nouvelles normes de vie au travail. Les résultats feront état du développement du pouvoir d'agir individuel et collectif au sein d'un comité santé. 


\section{INTERVENIR COLLECTIVEMENT SUR LE TRAVAIL QUI FAIT SOUFFRIR : ENTRE SOURCES DE TENSION ET DÉVELOPPEMENT DU POUVOIR D'AGIR D'UN COMITÉ SANTÉ À L'ÉCOLE}

INTERVENING COLLECTIVELY ON WORK RELATED SUFFERING : BETWEEN SOURCES OF TENSION AND POWER TO ACT OF A SCHOOL HEALTH COMITY

Emmanuel Poirel ${ }^{1}$ Université de Montréal
Marc-André Houde Université de Montréal

\section{SANTÉ MENTALE ET DÉTRESSE PSYCHOLOGIQUE CHEZ LES PERSONNELS SCOLAIRES}

Selon l'enquête québécoise des conditions de travail d'emploi et de santé-sécurité du travail, en comparaison aux autres professions, le milieu de l'éducation ferait partie des plus exigeants (Vézina, Cloutier, Stock, et al., 2011). Les salariés du secteur de l'enseignement seraient les plus atteints par la détresse psychologique, la violence physique au travail et la consommation de psychotropes. La Fédération des commissions scolaires du Québec, montre que près de la moitié des absences au travail sont la conséquence de problèmes liés à la santé mentale et le nombre d'absences du travail ne cesse de s'accentuer. La recherche, de Houlfort et Sauvé (2010), sur 2401 enseignants montre que près de la moitié vivent une baisse d'énergie tous les jours et près des deux tiers présentent des symptômes d'épuisement professionnel tous les mois. Même chose chez les professionnels (conseillers d'orientation, orthopédagogues, psychologues, conseiller pédagogique, etc.) où plus de la moitié sont en surcharge de travail et le tiers est à risque d'épuisement (Soares, 2004). Le personnel de soutien souffre également en raison du manque de ressources et de l'insécurité (Maranda et Viviers, 2011) et les directions ne sont pas en restes devant le stress de la fonction et les risques pour leur santé mentale (Poirel, Yvon, Lapointe et Denecker, 2017).

Sans négliger les caractéristiques individuelles, tout comme les particularités des types d'emplois (p. ex., enseignants, professionnels, soutien, direction), la recherche montre que les principales sources de difficulté viennent du climat dans l'école, de la violence, des dynamiques relationnelles (Jeffrey et Sun, 2006) et de la multiplicité des tâches administratives (p. ex., gestion des absences, collectes de fonds, rapports, formulaires). À titre d'exemple, on constate que les cinq principales sources de tensions au travail chez les enseignants sont la charge de travail, la gestion de classe, la relation avec la direction, les parents et les collègues (Houlfort et Sauvé, 2010), et chez les directions, les contraintes

1. Adresse de correspondance: Département d'administration et fondements de l'éducation, 90, avenue Vincent d'Indy, Montréal (QC), H2V 2S9. Téléphone : 514-3436413. Courriel : emmanuel.poirel@umontreal.ca 
administratives, les relations interpersonnelles, les conflits intrapersonnels, les responsabilités administratives et les attentes de rôles (Poirel et al., 2017) ainsi que les multiples changements qui se perpétuent (Gravelle, 2017).

Ainsi, ce serait sur ces sources qu'il faudrait intervenir pour tenter de contrer le phénomène et limiter les risques sur la santé mentale au travail de ces professionnels du secteur de l'enseignement. Or, force est de constater que chacun des milieux est unique et qu'il possède sa propre culture, son histoire, ses dynamiques sociales et interpersonnelles. Comme le laissent entrevoir les travaux de Maranda et Viviers (2011), il faut parfois changer l'angle de vue, trop souvent dirigé sur les personnes considérées comme incapables de s'adapter aux sources de difficultés. II faut davantage s'intéresser aux dynamiques sociales afin de permettre aux personnes concernées de travailler collectivement à confronter les conditions de travail qui les font souffrir. Une telle perspective invite à initier des approches d'intervention collectives, qui prennent en compte le contexte et la dynamique particulière du milieu concerné, qui seraient plus à même de prévenir à la source les problèmes liés à la santé mentale (Richard, Cherkaoui et Christin, 2015).

Devant cet état de fait en regard de la santé mentale et la détresse psychologique du personnel scolaire, on peut s'interroger sur ce qui a été fait pour améliorer cette situation et parvenir à enrayer ce mal. En d'autres mots, comment intervenir à la source de ce problème?

\section{SUR LES LIMITES DE L'INTERVENTION EN SANTÉ AU TRAVAIL}

L'Organisation mondiale de la santé (OMS) distingue trois types d'intervention pour contrecarrer les problèmes de santé (primaire sur les sources, secondaire sur la sensibilisation et l'éducation dirigée sur la personne et tertiaire plutôt curative). Issues de notre expérience de clinicien intervenant sur le terrain, nous évoquons certaines limites de l'intervention en matière de prévention de la santé mentale au travail en milieu scolaire afin d'en tirer leçons et de guider de futures actions pour l'intervention. Notons d'abord que les principales interventions sur la santé que l'on retrouve dans le domaine scolaire visent avant tout à outiller les personnes à faire face à leurs difficultés professionnelles. Ainsi, il s'agit principalement d'interventions de type secondaire (p. ex., développement de compétences professionnelles, augmentation des capacités de résistance au stress ou d'évacuation par la méditation pleine conscience, la résilience...), ou d'interventions de type tertiaire, lorsque les employés se retrouvent en détresse et dans l'incapacité de réaliser leurs tâches ( $p$. ex., programme d'aide aux employés). Or, sans nier leur efficacité pour diminuer les symptômes, ces approches tendent à individualiser le rapport 
entre santé mentale et travail. En rendant les personnes responsables de leur santé (et conséquemment de leurs problèmes), sous prétexte de les rendre plus capables, une telle perspective de la santé tend à les isoler sur elles-mêmes et peut avoir pour effet d'amputer le pouvoir d'agir sur le travail sans pour autant prévenir et agir à la source (intervention primaire) des problèmes de santé psychologique au travail (Harvey, Courcy, Petit, Hudon, Teed, Loiselle et Morin, 2006; Rhéaume, Maranda, Deslauriers, Saint-Arnaud et Trudel, 2008).

Si les interventions de type primaire sont plus rares (Cooper, Dewe et O'Driscol, 2001), cela semble pouvoir s'expliquer par le fait qu'agir au niveau primaire est plus coûteux en temps et en énergie comparativement aux interventions menées aux niveaux secondaire et tertiaire. L'intervention primaire implique de faire participer le collectif dans la remise en cause des conditions, des pratiques organisationnelles et de l'organisation du travail (Maranda, Viviers et Deslauriers 2014).

Sur la base de ces considérations, notre perspective de rechercheintervention s'appuie sur la conception que la santé au travail, tant individuelle que collective, se construit collectivement par l'actualisation et le développement du pouvoir d'agir des travailleurs sur les règles, normes et modes d'organisation du travail (Martin, 2016; Moussay et Méard, 2011); un pouvoir qui se mesure par la possibilité pour les enseignants, professionnels et directions, d'influencer leur contexte de travail et d'en faire un monde vivable, en générant de nouvelles normes de vie au travail (Canguilhem, 1966). Intervenir en considérant le pouvoir d'agir comme vecteur de santé mentale au travail implique par conséquent, une prise en charge collective de la santé au travail.

C'est donc en considérant ces limites que nous sommes intervenus dans une école secondaire de la grande région de Montréal, dans le cadre d'un projet de recherche-intervention sur la santé au travail, avec comme objectif général, de favoriser et de documenter le développement du pouvoir d'agir individuel et collectif dans la perspective d'une meilleure santé au travail. Dans cet article, après avoir présenté les fondements sur lesquels repose la recherche-intervention ainsi que son déroulement, nous présentons une vignette clinique qui témoigne des contraintes et du développement du pouvoir d'agir des membres du comité santé.

\section{LES FONDEMENTS DE LA RECHERCHE-INTERVENTION}

Précisons que nous nous sommes inscrits dans une démarche collaborative afin de permettre aux individus de participer collectivement tout autant à l'identification des sources pathogènes, qu'à la découverte de stratégies salutogènes. Notre approche de recherche-intervention participe 
à la vision renouvelée de la relation d'aide, de la formation et du développement des personnes en réinterrogeant les rapports entre l'observateur et l'observé. La recherche où l'intervention ne s'effectuent plus "sur », mais " avec » les sujets d'étude et elles se font "action » vers une transformation (Doucet et Bourassa 2016). Le paradigme de l'expert en intervention est ainsi remis en question, afin de laisser davantage d'espace, pour l'actualisation des capacités d'agir des personnes impliquées dans la démarche (Le Bossé, 2003; Paul, 2009). Et le savoir n'appartient plus seulement à la science et aux chercheurs, mais aussi aux praticiens, qui sont également considérés comme producteurs de connaissances (Perrenoud, 2012).

Ainsi, la recherche-intervention que nous avons menée s'inscrit dans la perspective où «les modalités d'action ne seraient plus à l'unique initiative du chercheur, ni commandées par une institution, mais seraient soumises à négociation » (Mérini et Ponté, 2008, p. 79). Elle s'appuie sur les présupposés de la théorie de l'activité (Engeström, 2000) qui postule que le travail ne fait pas partie d'un contexte extérieur qui affecte l'être humain, mais plutôt qu'il est la source du développement humain. Comprendre un tel développement implique de s'intéresser à l'activité humaine, envisagée comme une activité socialement située dans son contexte et son histoire. Notre approche s'inspire aussi de la méthodologie particulière de l'autoconfrontation en clinique de l'activité (Clot, Faïta, Fernandez et Scheller, 2001). Nous postulons que les travailleurs euxmêmes sont les experts de leur activité (Clot et al., 2001; Yvon et Saussez, 2010) et que "seuls les collectifs eux-mêmes peuvent opérer des transformations durables de leur milieu de travail " (Clot et al., 2001, p. 170). Nous considérons qu'intervenir pour prévenir les situations à risque pour la santé en milieu scolaire, s'inscrit dans un processus social d'intercompréhension sur les enjeux de santé individuelle et collective, ce qui implique de façon interdépendante l'ensemble du personnel et des directions d'établissement (Rowling et Samdal, 2011). C'est-à-dire que le développement du pouvoir d'agir, à la fois individuel et collectif, se manifeste dans la relation constante qui existe entre les "possibilités d'action " externes à la personne (p. ex., dynamiques sociales, culture organisationnelle, participation aux décisions, accès aux ressources et aux activités de développement professionnel, contexte politique et législatif, routines organisationnelles, etc.) et les "capacités d'agir " sur les composantes du travail. Ces capacités d'agir sont considérées comme des ressources internes à l'échelle individuelle (p. ex., compétences, motivation, sentiment d'efficacité personnelle, niveau de conscience, émotions) ou à l'échelle collective (p. ex., comité dans l'école) (Gouédard et Rabardel, 2012; Le Bossé, 2003). 
C'est donc sur cette base que nous avons mené cette rechercheintervention (deux chercheurs cliniciens), avec un double rôle étant à la fois clinicien, de par notre expertise de terrain sur l'intervention en santé au travail, et comme chercheur, pour documenter les retombées de l'intervention.

\section{CONTEXTE ET DÉROULEMENT DE LA DÉMARCHE DE RECHERCHE- INTERVENTION SUR LA SANTÉ AU TRAVAIL}

L'intervention que nous avons menée s'inscrit dans une démarche de qualité de vie au travail (QVT), qui s'est déroulée entre les mois d'août 2016 et de juin 2017, dans une des plus grandes écoles secondaires du Québec en contexte urbain. L'école était aux prises avec des changements importants et la direction était préoccupée par la santé mentale de son personnel (retour de classes régulières après 8 ans d'absence, accueil de 40 nouveaux membres du personnel et de plus de 200 élèves, travaux de rénovation, changement de locaux).

La démarche s'est déroulée selon la séquence suivante :

1. Août 2016 - Rencontre (deux chercheurs cliniciens) avec la direction.

2. Septembre 2016 - Rencontre avec la direction et ses cinq adjoints pour apprécier leur réalité, présenter et s'entendre sur les objectifs et modalités de la démarche et élaborer un objectif général d'intervention.

3. Septembre 2016 - Conférence sur la santé mentale au travail destinée à l'ensemble du personnel et invitation à faire partie d'un comité santé (le comité a été constitué de la direction, de cinq enseignants et d'une technicienne en éducation spécialisée).

4. Octobre 2016 - Invitation à tous les membres du personnel de l'école à compléter un questionnaire de santé globale au travail (QSG), comprenant les sources de stress et les contraintes au pouvoir d'agir afin de dresser un portrait de la situation quant à la santé au travail de l'ensemble du personnel de l'école. Sur 135 répondants, nous avons obtenu les réponses de 91 enseignants; 28 personnels de soutien, sept professionnels et cinq directions (quatre répondants n'étant pas identifiés). L'analyse nous a permis de faire certains constats que nous avons présentés dans un rapport, en premier lieu à l'équipe de gestionnaires, avant de le porter à débat avec le comité santé lors des rencontres de travail.

5. Novembre 2016 et février 2017 - Deux premières rencontres du comité santé pour identifier, discuter, débattre et sélectionner les principales sources de tensions dans l'école sur lesquelles agir, en s'appuyant sur les résultats du questionnaire. 
6. Mars à juin 2017 - Trois autres rencontres du comité pour élaborer des solutions, une politique de santé et bien-être au travail et proposer un plan d'action.

\section{DÉROULEMENT DE LA DÉMARCHE MÉTHODOLOGIQUE}

Afin de répondre à notre objectif de favoriser et de documenter le développement du pouvoir d'agir individuel et collectif dans la perspective d'une meilleure santé au travail et pour répondre à cette double finalité de recherche (production de connaissances) et d'intervention (transformation), nous avons utilisé un dispositif méthodologique provenant du courant ergonomique de l'analyse du travail (Yvon et Garon, 2006). Sur cette base, nous avons adapté à nos besoins de recherche le dispositif d'autoconfrontation vidéo simple (ACS) avec quatre membres du comité santé et d'autoconfrontation vidéo collective (ACC) avec la totalité des membres.

Les membres du comité santé ont donc été filmés durant leurs rencontres de travail dans lesquelles étaient discutés les résultats des questionnaires santé dans la perspective d'identifier et de proposer des solutions pour l'établissement. Après chacune des rencontres d'une durée de 90 minutes, nous (chercheurs cliniciens) avons identifié sur les vidéos entre 6 et 10 situations considérées comme des contraintes et des possibilités de développement du pouvoir d'agir. Pour chacune de ces situations, nous avons effectué un montage vidéo d'une durée moyenne d'une minute que nous avons présenté à tour de rôle aux participants du comité santé en confrontation vidéo simple. Plus concrètement, la procédure a consisté à confronter individuellement, en alternance, quatre membres du comité santé ayant participé aux réunions filmées dans une entrevue semi-dirigée de 60 à 90 minutes, à la suite des rencontres de travail. Parmi les sept membres du comité santé, quatre ont volontairement accepté de participer aux ACS, la direction et trois enseignants. Les participants ont donc été confrontés aux images vidéo de leur travail sur les situations identifiées, en lien avec leur pouvoir d'agir, qui leur étaient présentées. Appartenant à la famille du rappel stimulé (Tochon, 1996), les situations ont permis de solliciter les commentaires individuels sur les situations vécues. Au final, nous avons obtenu 4.5 heures de captation vidéo du travail réel des travaux du comité santé, à partir desquelles, nous avons identifié 28 séquences considérées comme des contraintes et des possibilités de développement du pouvoir d'agir.

Par la suite, nous avons identifié six situations parmi les 28 que nous avons considérées comme les plus emblématiques selon trois critères (la récurrence, l'intensité et le développement du pouvoir d'agir): 1) les assemblées générales, 2) le temps et la libération des enseignants, 3) les 
dîners entre les enseignants et la direction, 4) les attentes et la disponibilité des directions, 5) les structures et les routines, 6) les leaders émergeants en santé au travail.

Ces séquences ont été présentées en entretien de confrontation vidéo collective avec l'ensemble des membres du comité santé lors d'une journée pédagogique. Durant cette rencontre, les situations étaient présentées afin que les collègues soient invités à s'interroger sur leurs manières de faire, ce qui avait pour but d'enrichir collectivement les commentaires sur les manières de faire. Précisons que les rencontres de confrontation vidéo simple et la confrontation vidéo collective ont également été filmées pour fin d'analyse, et que l'ensemble du matériel (4.5 heures en temps réel et 6 heures en confrontation vidéo) a été transmis en verbatim avant d'être analysé à l'aide du logiciel QDA Miner.

Le rôle des chercheurs cliniciens a donc été celui d'accompagner les participants lors des rencontres de travail en comité, lors des confrontations vidéo simples et lors de la rencontre de confrontation vidéo collective. En contrôlant le défilement des images et en posant des questions de relance sur le contenu de l'image dans l'exploration des conflits et des dissonances de l'activité, l'objectif était de permettre aux sujets de s'exprimer ouvertement sur leur vécu en lien avec l'enregistrement vidéo, et ce, en vue de favoriser le développement potentiel de l'activité de travail et du pouvoir d'agir, du comité santé dans l'école et de ses membres dans le comité santé et l'école. Précisons que lors de la rencontre de confrontation collective, les membres du comité santé ont aussi eu l'occasion de partager ce rôle avec les chercheurs cliniciens en posant des questions entre eux, favorisant du coup, de nouvelles possibilités de transformation du travail avec le collectif.

Spécifions aussi, sur le plan méthodologique, que si l'observation directe captée sur vidéo permet une analyse en profondeur d'une situation problématique, elle ne donne cependant pas accès aux pensées (cognitions) des acteurs en situation. Or, les ACS et ACC donnent un accès privilégié aux cognitions situées des acteurs au cœur de leur activité. À titre d'exemple, pour une séquence d'une minute d'activité réelle observée en ACS, on obtient entre 15 et 30 minutes de retour du participant, à qui l'on demande de répondre à la question « que se passe-til dans ta tête à ce moment précis? "

C'est à la lumière de ce constat que nous présentons ici une des cinq vignettes cliniques issues de notre analyse, et qui témoignent du développement du pouvoir d'agir, des membres du comité santé ayant participé à la démarche de qualité de vie au travail. 


\section{RÉSULTATS : PRÉSENTATION ET ANALYSE D'UNE VIGNETTE CLINIQUE SUR LES CONTRAINTES DE TEMPS ET LA LIBÉRATION DES ENSEIGNANTS}

La situation que nous présentons, concerne les contraintes de temps que vivent les enseignants membres du comité santé qui désirent s'investir dans la démarche de QVT, mais qui sont confrontés à devoir se faire remplacer dans leur classe par un suppléant. Si la solution proposée par la direction semble à première vue évidente, il apparaît que dans la réalité du travail, elle tend plutôt à créer une contrainte au travail bien fait des enseignants.

La séquence est présentée en trois temps 1) le réel de l'activité dans lequel la direction (D) offre aux enseignants d'être libérés afin de pouvoir travailler en comité sur la santé; 2) la confrontation vidéo simple de cette situation présentée qui permet de rendre compte de la " cognition » d'un enseignant (E5) devant cette solution proposée et 3) la confrontation collective de la situation qui ramène le débat sur cette contrainte, donne accès aux cognitions situées et favorise ultimement le développement du pouvoir d'agir.

Possibilités d'action : occasions et libérations pour la réalisation des tâches du comité

Au début de la démarche en septembre 2016, un temps de libération avait été alloué et réparti sur l'année par la direction pour le travail dans ce comité santé. Or, il est vite apparu en cours de route de la démarche que le temps était un enjeu important, pour les membres du comité. Le comité avait comme tâche non seulement de prendre connaissance des problèmes en appui des résultats au questionnaire santé, afin de proposer des solutions à l'ensemble de l'école en vue d'un plan d'action et de l'élaboration d'une politique santé, mais aussi de consulter au fur et à mesure leurs collègues dans l'ensemble de l'école ainsi que de présenter les avancements de leurs travaux.

La situation que nous présentons concerne les tâches et responsabilités des enseignants en regard du comité santé en lien avec le manque de temps. Dans la situation, la direction propose une date où tous les membres du personnel de l'école sont présents et disponibles, afin que le comité santé puisse présenter la démarche et l'avancement des travaux du comité, à tous les membres du personnel de l'école. La proposition de la direction est fortement appuyée par l'un des deux chercheurs intervenants, qui en profite pour encourager l'équipe dans cette voie en offrant de l'aide, mais en insistant sur la prise de responsabilité des membres du comité. Or, la réaction des membres fait clairement état d'une réticence marquée, d'un frein à s'engager dans cette voie. 
Comité santé en rencontre de travail filmé en temps réel :

Direction: " pour pouvoir diffuser cette information, on pourrait se servir de ce moment-là. Nous, on a 15 minutes...c'est la dernière journée d'étape, ce n'est pas le temps de les submerger. Mais si vous voulez comme comité utiliser ça, je vais avoir tout le monde devant moi là "

Chercheur : " moi je pense que c'est une excellente idée pour faire le bilan des avancements des travaux du comité santé. C'est vous qui allez prendre la parole. On peut vous aider là-dedans, mais c'est pas nous qui allons le faire. On peut vous aider à construire un PowerPoint. On peut mettre toutes les choses en place et vous, vous avez à livrer le message »

Enseignant (3) : "parce que nous on est dans notre remise des notes ».

Direction: "contraintes de temps là. Si vous voulez que je vous libère une journée pour le faire, c'est correct. C'est prioritaire là ». "Le comité qui est là pourrait devenir une forme de comité... euh projet éducatif... euh projet santé globale euh... un projet pilotage de l'école... y faut y mettre l'énergie et reconnaître. Je sais que vous haïssez ça vous faire libérer, mais je l'ouvre, cela ne me dérange pas. Enseignant (1): Ben c'est-tu un prof de maths qui va me remplacer? Sinon c'est non, catégorique. Si ça peut être un prof de maths, oui. Mais sinon... C'est parce qu'on est en plein fin d'étape. C'est un timing qui est très serré. Là mes élèves je peux pas les laisser, c'est un cours de perdu.

Cognition située d'un enseignant (5) appréhendée lors de la présentation de cette séquence en confrontation vidéo simple :

Chercheur: "on vous annonce que vous avez la possibilité de faire une présentation. Là on te voit réfléchir à ce moment-là. Tu penses quoi à ce momentlà, quand on te dit: "oups, tu vas aller présenter devant tes collègues, la démarche? "

Enseignant (5) : Ben, j'ai pensé : " est-ce que je suis prêt? Donc, c'est ça ma préoccupation. Je voyais, est-ce qu'on est...on est tu prêt pour faire ça? Je ne sais pas... Y'a une inquiétude.

Chercheur: Après ça, un peu plus loin, l'autre séquence qui vient c'est : " on peut vous libérer. On peut vous donner du temps. "

Enseignant (5) : À ce moment-là, je dis OK : "oui c'est vrai. " Je dis : " c'est bien, il tient à cœur qu'on puisse se libérer. ". OK, j'ai dit: "OK, il est prêt à donner du temps pour se préparer, c'est bon tout ça. Ouais. Mais c'est surtout là que des fois c'est comme, la fin d'étape... ça fait comme une surcharge parce qu'on se rencontre jeudi soir pour préparer ça, le lendemain... Là y'avait comme une surcharge là.

Chercheur: Même si on libère, y'a quand même cette inquiétude.

Enseignant (5) : Oui. Mais parce que, quand on y va, on ne voit pas notre groupe, c'est ça l'affaire... c'est...aussi long préparer une...quelqu'un qui va remplacer, qu'est-ce qui va faire, pis tout ça, donc...préparer la suppléance, pis tout ça... Ce n'est pas si aidant que ça.

Chercheur: Y'a tout ça qui te passe dans la tête.

Enseignant (5) : Oui, oui, oui, oui. C'est la gestion du temps là... ça va trop vite, est-ce qu'on peut attendre peut-être la semaine d'après. Une fois que les notes sont remises, c'est comme " ouuuuu » [en signifiant une baisse de pression]. C'est 
toujours incroyable, mais c'est toujours un rush à la fin là...hein [rire]. Après ça, c'est comme " puff »...Tout le monde est plus détendu.

Chercheur : Comment tu perçois ça là, le fait que lui propose de vous libérer?

Enseignant (5) : Moi je dis OK: "wow, c'est bien là...il sort...un budget pour..." Je ne sais pas s'il doit en avoir un budget. Mais, c'est bien, il va de l'avant là : " il faut que ça fonctionne, il faut... ". C'est le timing! C'est le timing, juste ça. Parce que je dis : "si on se fait libérer par après. "...on est prêt et disposé à écouter ce qu'ils ont à nous proposer. Pis on n'est pas comme: "OK, je dois aller dans ma classe après, pis tout ça. ".

Cognitions situées des enseignants et de la direction appréhendée lors de la présentation de cette séquence en confrontation vidéo collective :

Chercheur: [Visionnement de la vidéo] Qu'est-ce qui se passe à ce moment-là dans votre tête?

Enseignant (1): Faque là, on dit: "ben oui, je vais être...libéré ». Ça ne me dérange pas d'être libéré si mes élèves ont des conditions acceptables. C'est pas vrai que je vais être libéré pour que les élèves s'en aillent à la cafétéria. C'est ça qui se passe dans ma tête. Pis c'est pas [pointant la direction]... il manque de suppléants. C'est notre réalité. C'est pas vrai qu'en maths de 5 je vais perdre ma période. Ça ne se fait pas. Donne-moi un prof de maths, pis je vais participer. Mais, d'abord et avant tout, mes élèves il faut qu'ils soient bien. Faque oui j'aime ça être libéré, si les conditions sont bonnes. "Ah, j'ai le goût. J'ai le goût. Mais trouve-moi un prof de maths OK? "C'est ça qui se passe dans mon cerveau ».

Enseignant (3): Moi, je regarde mon agenda. Je me souviens très bien. Parce que là, je suis dans ma tête là, pis je planifie là. Pis moi, vendredi, il faut que mes notes rentrent là. Pis là, je suis comme: "OK. Mais là là. OK. Là, j'ai telle période. J'ai un trou là. J'ai de la correction. J'ai un examen jeudi. Donc, il faut qu'il soit corrigé pour vendredi. " Là, je calcule moi. Je suis en train de calculer. Et je réagis quand... M. Directeur nous dit: "ben, je sais que vous haïssez ça vous faire remplacer ", parce que c'est vrai que c'est pas productif du tout. Parce que moi j'ai des textes, je donne des textes, mais souvent les textes faut qu'ils soient analysés avec les élèves. C'est pas le suppléant qui va faire ça...Moi, j'ai l'impression de...que j'augmente ma charge de travail au lieu de... Et je...pour moi, c'est pas utile de me faire dégager de mes tâches...Parce qu'à un moment donné, je n'arrive plus dans mon temps. Là... ça ne va pas bien.

Enseignant (5): Moi c'est un petit peu comme lui [E3] aussi. C'est...sûr que se faire remplacer c'est...se faire libérer, mais après ça là, c'est ...ça s'enchaîne un petit peu de retard.

Enseignant (2): Moi, c'est...j'avais pas...j'étais très mal à l'aise avec le peu de délais. Je suis une personne qui aime planifier. Faque, moi aussi quand j'ai planifié, faire tout ce changement-là ça me cause un stress. Ça me stressait. Ça me stressait à l'idée de me faire remplacer. Moi aussi je suis en fin d'étape, puis j'ai une situation à gérer avec les élèves. Pas juste une préparation didactique, mais une préparation mentale aussi. Se sentir prêt. Moi c'est un tout pour moi. Je trouvais les délais trop...serrés pour toutes ces raisons. Puis, y'avait une question aussi, je pensais à mes collègues, ça ne serait pas bien reçu de faire une présentation quand on leur dit: " on travaille sur...reconnaître la complexité de votre tâche. On le sait que vous êtes dans un rush. Mais là, on va vous faire une petite présentation au lieu de vous permettre de vous libérer pour faire vos notes. " 
Faque moi je trouvais qu'on allait à l'encontre de ce qu'on dit, disons, à nos équipes de travail. On essaie de faire réaliser [accent mis sur le mot] la complexité de la tâche. Faque c'était comme pas cohérent pour moi.

Enseignant (4) : ...pis c'est un petit peu là ce que E2 a dit...je me dis... Pis c'était aussi dans la même optique on dit aux gens: "on veut que vous soyez bien à l'école... " et là on va arriver, fin d'étape, où on le sait qu'on étouffe tous à ce moment-là. Nous on va arriver et on va dire: "il va avoir ça. " Moi c'était le commentaire que j'avais fait hein, un peu à tout le monde. Je ne me sentais pas bien là-dedans.

Direction: Moi je cherchais une solution. Moi je sentais une volonté: "faut partager ça là! ॥ Bon, ben. De toute façon... c'était pas une bonne idée. J'ai pas pensé, c'est drôle hein, j'ai pas pensé fin d'étape quand j'ai... J'ai pas du tout pensé à ça... Moi ma fin d'étape en fait...mon rush arrive tout de suite après vous autres. ...et je voulais, un moment donné, vous dire: "regardez là. Si c'est trop intense ce comité-à, j'ai une ressource, je peux vous aider. "... Bon, pis y'a des contraintes syndicales parce que...ce que tu viens de...ce qu'on disait au point précédent (suppléant de math), ben moi aussi là, je ne peux pas prendre qui je veux. J'ai...des règles qui m'obligent à prendre des gens spécifiques... T'sais. Un moment donné... Mais je voulais au moins vous dire ça, vous dire: "regardez là, je suis prêt à bien des affaires. J'ai les ressources. " Bon, il faut savoir les utiliser. C'était ça le message que je voulais vous transmettre d'abord.

\section{Conclusion de la rencontre de confrontation collective qui témoigne du développement du pouvoir d'agir}

Chercheur: Dernière chose. Je ne veux pas l'oublier. Mais la dernière rencontre c'est le 29 mai...

Direction : Ben, en fait l'objectif de la prochaine rencontre va être de planifier... Toujours les solutions, mais aussi de planifier...notre finale...

Enseignant (3): Oui. Oui. C'est ça.

Enseignant (4): Oh oui, pour...l'Assemblée là.

Direction : Parce qu'on vient de se...on a 2 volets... Pis on pourra continuer dans les solutions l'année prochaine...

Chercheur: On va prendre le temps qu'il faut. L'important c'est qu'on aille déjà des petites solutions qu'on mette en place avant de proposer un plan.

Enseignant (3): C'est ça. De toute façon, E4 et moi, on n'a pas fini nos consultations.

Enseignant (4): Ben, je ne le sais pas. Tantôt vous parliez de libération [en pointant la direction].

Direction : Ouais. Je continue.

Enseignant (4): Non, mais, moi je me dis, s'il y a lieu, non, mais dans les sessions d'examen, on n'a plus de planification, on n'a plus de... On a notre rencontre du 29 mai, mais admettons qu'on veut, t'sais, admettons que le 29 on termine les points qu'on n'a pas eu le temps de voir la dernière fois, pis on a besoin de temps. Sans que ce soit fait, bon pendant les cours...ben peut-être que ça pourrait être une possibilité pendant les sessions d'examens.

Enseignant (3) : Moi je trouve que c'est une bonne idée.

Enseignant (4): On pourrait être libéré de surveillance, mais on a une rencontre et là on aurait peut-être même un petit peu plus de temps. Un après-midi t'sais. 
Direction : Ben en fait, c'est vous 2 [E1 et E4]. Toi [E4] pis toi [E1], pis toi [E2] t'en as aussi pas mal...Mais...oui, oui. Il faudrait que je regarde le moment où ça ne nuit pas à personne...Faque, donc je mets des suppléants, j'en ai déjà partout là t'sais. C'est un moment où... Ben là, parce que je pense à votre planification. Dans l'idée : "oui, mais pas trop déranger vos affaires. "

Chercheur : C'est quand votre présentation?

Direction : Le 26. Moi je pense qu'on va faire ça le 26 juin. C'est la dernière journée... On a une Assemblée générale où justement on se raconte nos bons coups. Faque c'est parfait là

Enseignant (3) : Faque oui. Ça serait la bonne...

Direction : Faque je prévois une journée de libération. Dans le fond, le 29 mai on va s'en servir pour travailler sur les solutions santé qu'on...OK...Et je prévois une journée de libération pour qu'on prépare le spectacle.

Enseignant (4): Pendant la session d'examens.

Direction : Pendant la session d'examens si je suis capable.

Enseignant (3) : Ouais. Ouais. Ouais. Ça serait une bonne idée.

Direction: J'essaie de trouver un lieu où vous n'aurez pas la responsabilité d'épreuve...

L'analyse de cette situation observée dans le réel de l'activité des enseignants et de la direction, ainsi que les contenus de pensée (cognitions) accessibles par confrontation vidéo, montre l'ampleur des enjeux. Si la direction cherche des solutions afin de permettre aux membres du comité santé de s'engager et que la libération des enseignants peut sembler la plus évidente des solutions, la réalité montre que cette solution est davantage perçue comme une contrainte qui peut être interprétée comme une limite à leur pouvoir d'agir sur leur travail.

Notons d'abord, en regard de la santé mentale, que le court délai proposé est perçu par les enseignants comme une inquiétude et une source de tension, quant à la qualité du contenu qui sera présenté à leurs collègues sur les avancements des travaux du comité santé. Mais aussi, on peut constater que l'inquiétude dépasse le travail en comité, dans la mesure où se faire remplacer implique une crainte de la diminution de la qualité dans leurs enseignements et le progrès de leurs élèves, tout comme une entrave à l'ensemble des tâches que les enseignants ont à accomplir en dehors de la classe, par exemple la préparation des cours et les corrections en période de préparation des bulletins.

On peut également constater que la rencontre de confrontation vidéo collective, qui ouvre le débat sur cette source de difficulté, finit par faire aboutir à la découverte de nouvelles solutions en tenant compte des réalités de chacun; les contraintes des enseignants certes, mais aussi de la direction (syndicales, pénurie de suppléants, augmentation de la charge de travail non prescrite des concierges, locaux, etc..). Les ressources sont là et la direction est prête à investir, mais les enseignants doivent pouvoir y trouver leur compte, c'est-à-dire en mesurer les retombées positives sur le 
plan individuel. Au final, la solution qui émerge semble être une meilleure planification et une libération pour les enseignants dans des périodes qui n'influent pas sur la qualité de leur enseignement, des périodes moins achalandées dans le rythme cyclique d'une année scolaire normale.

Ce qui ressort aussi de l'analyse, c'est que cette décision s'est prise dans la collaboration entre les acteurs qui ont trouvé le moyen de dépasser les contraintes du travail en acceptant mutuellement les réalités de chacun. C'est en prenant part à des tours de paroles et en étant ouvertement confrontés aux contraintes de chacun que s'est dessinée une solution commune. C'est dans ce débat d'ouverture, de dialogue tolérant et d'écoute risquée que chacun a pris du pouvoir sur son milieu.

On constate également vers la fin de la rencontre de confrontation collective un changement de ton et d'ambiance. Si le début de la rencontre est plus tendu, et à raison, dans la mesure où était présentée une source de tension partagée, la solution qui émerge tend à favoriser une reconstruction collective de l'activité de travail plus optimiste. Le fait pour la direction à la fin de la rencontre, de parler de la présentation comme d'un "spectacle », n'est aussi pas banal et fort de symbolique, dans la mesure où elle veut marquer le pas, en donnant envie aux autres membres du personnel de l'école de s'engager dans cette démarche. Le comité passe à un autre niveau d'engagement qui témoigne du développement d'un pouvoir d'agir individuel et collectif.

\section{DISCUSSION ET CONCLUSION}

Intervenir sur les difficultés vécues par les enseignants afin de prévenir les risques pour leur santé mentale implique d'inscrire la santé au travail dans ses dimensions individuelles et collectives en dépassant une vision où la santé serait uniquement une responsabilité individuelle. La santé mentale au travail dans un milieu aussi complexe que l'éducation implique de considérer les dynamiques sociales en jeu, et dans cette perspective, d'intervenir de façon collective sur les sources de difficultés vécues.

Les interventions de niveau secondaire (éducation) et tertiaire (curative), malgré leur efficacité, sont destinées à permettre aux travailleurs de mieux s'adapter à leur travail, et dans ce sens, ne permettent pas d'agir sur l'organisation du travail et ses conditions qui font souffrir (Harvey et al., 2006; Rhéaume et al., 2008).

Nos résultats montrent qu'en mettant en place une approche d'intervention centrée sur les sources (primaire) et en faisant participer le collectif (équipe-école et direction), tant dans la recherche des causes que dans celles des solutions, il s'avère possible de renverser cette 
perspective. C'est-à-dire qu'en mettant à débat les difficultés dans un climat d'ouverture et de dialogue, l'intercompréhension des difficultés de chacun qui s'en dégage, plutôt que de permettre aux personnes de s'adapter de façon individuelle et souvent isolée, favorise une transformation du travail qui finit, «lui », par s'adapter à la réalité psychologique des travailleurs.

Les résultats de notre intervention dans une école secondaire vont dans ce sens. En identifiant collectivement les sources de difficultés vécues tout en permettant aux enseignants de participer à réorganiser leur travail à l'échelle collective, sur la base de ces sources a permis de bonifier l'organisation du travail, en prenant en compte la singularité du milieu, dans la perspective d'une meilleure santé mentale au travail (Brun et al., 2007; Maranda et al., 2014).

Considérant la santé mentale dans son essence dynamique qui se construit par l'actualisation et le développement du pouvoir d'agir des enseignants sur leur milieu et qui dépasse nécessairement les limites de la classe, il faut y comprendre l'importance de la possibilité d'influencer le contexte et l'organisation du travail. Comme nous l'avons constaté dans notre démarche de recherche-intervention, en favorisant chez les enseignants, tout comme chez la direction et les autres professionnels, la possibilité d'agir sur le travail tout en respectant les capacités d'agir de chacun, cela a permis de générer de nouvelles normes, de nouvelles conditions de travail (Canguilhem, 1966). Mais aussi, dans la perspective d'un leadership renouvelé vers et pour la santé mentale au travail (Poirel, 2014), la prise en charge collective de la santé au travail a permis de faire émerger des solutions adaptées à la réalité singulière de leur milieu, en favorisant le pouvoir d'agir des enseignants sur les règles, normes et modes d'organisation, sur les conditions du travail collectif et conséquemment sur leur bien-être (Martin, 2016).

Dans notre démarche, en considérant le pouvoir d'agir comme un vecteur de la santé mentale au travail et les sources de tensions au travail du personnel scolaire comme des contraintes au pouvoir d'agir, l'approche clinique préconisée (Clot et al., 2001), dans une perspective collaborative (Doucet et Bourassa 2016), semble avoir permis aux membres du comité santé de surpasser ces contraintes et de transformer leur travail collectivement en développant leur capacité d'agir sur leur milieu (Moussay et Méard, 2011). Sur ce point, la confrontation collective semble avoir été un moment particulièrement riche en ce sens dans la mesure où la place et l'espace étaient disponibles pour l'actualisation et le déploiement des capacités d'agir des personnes (ressources internes). C'est en faisant équipe que les savoirs et l'expérience de l'un et de l'autre ont permis l'émergence de nouvelles solutions (Doucet et Bourassa 2016). 
À la lumière de ces résultats de recherche, il apparaît que les participants à cette démarche ont su bénéficier d'une augmentation de leur pouvoir d'agir, à la fois individuel et collectif qui, nous pouvons le supposer, a eu un impact sur leur santé mentale (Roche, 2014). C'est dans la dynamique intercompréhensive des difficultés vécues, de part et d'autre, que ce sont partagées des "possibilités d'action " collectives et des " capacités d'agir » individuelles (Gouédard et Rabardel, 2012; Le Bossé, 2003).

Si une telle démarche de recherche-intervention se distingue des interventions "classiques " fondées sur une vision "prescriptive " du changement et de l'organisation du travail, elle est nécessairement plus coûteuse en temps et en énergie. Mais, comme le montrent Rowling et Samdal (2011), une école vraiment promotrice de santé, doit nécessairement prendre le temps et les moyens pour favoriser une réelle distribution de l'influence au sein des différents acteurs de l'école, qui s'effectue lors d'un processus social d'intercompréhension sur les enjeux de santé individuelle et collective.

Notre démarche comporte toutefois certaines limites. Le nombre restreint de participants et la méthodologie déployée rendent les résultats difficilement généralisables à d'autres écoles. Aussi, l'impact des intervenants cliniciens-chercheurs sur l'ensemble de la démarche n'a pas été documenté alors qu'il ne peut être négligé. À cet égard, l'approche d'accompagnement d'un comité dédié à la santé au travail est habituellement initiée par un psychologue du travail et des organisations. Or, dans le cadre d'un comité santé en milieu scolaire, il faut y voir une opportunité pour les psychologues scolaires de favoriser en parallèle leur propre développement professionnel et conséquemment de participer à la promotion d'école vraiment promotrice de santé pour tous.

\section{RÉFÉRENCES}

Brun, J. P., Biron, C. et Ivers, H. (2007). Démarche stratégique de prévention des problèmes de santé mentale au travail, Rapport R-514. Montréal, QC : IRSST.

Canguilhem, G. (1966). Le normal et le pathologique. Paris, France : Presses universitaires de France.

Clot, Y., Faïta, D., Fernandez, G. et Scheller, L. (2001). Les entretiens en autoconfrontation croisée : une méthode en clinique de l'activité. Éducation permanente, 147, 17-25.

Cooper, C. L., Dewe, P. J. et O'Driscoll, M. P. (2001). Organizational stress. A review and critique of theory, research, and applications. Thousand Oaks, CA : Sage Publications.

Doucet, C. et Bourassa, B. (2016). Couleurs de la posture et des pratiques d'accompagnement en intervention et en recherche. Dans B. Bourassa et M.-C. Doucet (dir.), Éducation et vie au travail: Perspectives contemporaines sur les pratiques d'accompagnement pour l'orientation et l'intégration socioprofessionnelle (p. 1-8). Québec, QC : PUL.

Engeström, Y. (2000). Activity theory as a framework for analyzing and redesigning work. Ergonomics, 43(7), 960-974. 
Gouédard, C. et Rabardel, P. (2012). Pouvoir d'agir et capacités d'agir : une perspective méthodologique? Perspectives interdisciplinaires sur le travail et la santé, 14(2), 1-35.

Gravelle, F. (2017). « Impact d'un changement de gouvernance sur la profession de direction d'établissement d'enseignement œuvrant en milieu scolaire québécois et de direction dirigeant des écoles franco-ontariennes ", Recherches et éducations. Repéré à http ://journals.openedition.org/rechercheseducations/5273

Harvey, S., Courcy, F., Petit, A., Hudon, J., Teed, M., Loiselle, O. et Morin, A. (2006). Interventions organisationnelles et santé psychologique au travail, une synthèse des approches au niveau international. Rapport, Études et recherches, R-474. Montréal, QC : Institut de recherche Robert-Sauvé en santé et en sécurité du travail (IRSST)

Houlfort, N. et Sauvé, F. (2010). Santé psychologique des enseignants de la Fédération autonome de l'enseignement. Québec, QC : École nationale d'administration publique.

Jeffrey, D. et Sun, F. (2006). Enseignants dans la violence. Québec, QC: Presses de l'Université Laval.

Le Bossé, Y. (2003). De l'"habilitation" au "pouvoir d'agir" : vers une appréhension plus circonscrite de la notion d'empowerment ". Nouvelles pratiques sociales, 16(2), 30-51.

Maranda, M. F. et Viviers, S. (2011). L'école en souffrance : psychodynamique du travail en milieu scolaire. Québec, QC : Presses de l'Université Laval.

Maranda, M. F., Viviers, S. et Deslauriers, J. S. (2014). Prévenir les problèmes de santé mentale au travail : contribution d'une recherche-action en milieu scolaire. Québec, QC : Presses de l'Université Laval.

Martin, S. (2016). Le bien-être des élèves : pouvoir d'agir des enseignants débutants. Master Métiers de l'Enseignement de l'Education et de la Formation. Mention Enseignement Premier Degré. École Supérieure du Professorat et de l'Education de l'Académie de Nantes.

Mérini, C. et Ponté, P. (2008). La recherche-intervention comme mode d'interrogation des pratiques. Savoirs, 16(1), 77-95.

Moussay, S. et Meard, J. (2011). Le travail enseignant: une ressource pour le développement du pouvoir d'agir des enseignants débutants Colloque international INRP, 16, 17 et 18 mars 2011 Le travail enseignant au XXle siècle Perspectives croisées : didactiques et didactique professionnelle.

Perrenoud, P. (2012) "Développer la pratique réflexive dans le métier d'enseignant " (5 éd.). Issy-Les-Moulinaux, France : Éditions sociales françaises.

Poirel, E. (2014) Plaidoyer pour un leadership distribué favorisant la santé au travail en contexte scolaire. Qualaxia, 6, 12.

Poirel, E., Yvon, F., Lapointe, P. et Denecker, C. (2017). La fonction de direction scolaire adjointe : une comparaison des sources de stress entre adjoints et directions. Revue des sciences de l'éducation, 43(2), 231-260. doi :10.7202/1043031ar.

Roche, P. (2014). Normativité, grande santé et persévérance en son être. Perspectives interdisciplinaires sur le travail et la santé, 16(1), 1-12.

Rowling, L. et Samdal, O. (2011). Filling the black box of implementation for health-promoting schools. Health Education, 111(5), 347-366.

Rhéaume, J., Maranda, M.F., Deslauriers, J.S., Saint-Arnaud, L. et Trudel, L. (2008). « Action syndicale, démocratie et santé mentale au travail ». Nouvelles pratiques sociales, 20(2), 82-110.

Richard, D., Cherkaoui, W. et Christin, J. L. (2015). Des espaces de discussion pour articuler performance et santé au travail. Dans L. Karsenty (dir.), Quel management pour concilier performances et bien-être au travail? (Hors-série). Toulouse, France: OCTARĖS.

Soares, A. (2004). Travailler avec le coeur : la santé mentale, les émotions et les violences. Centrales des enseignants du Québec. Repéré à http ://www.fsq.csq.qc.

Tochon, F.V. (1996). Rappel stimulé, objectivation clinique, réflexion partagée. Fondements méthodologiques et applications pratiques de la rétroaction video en recherche et en formation. Revue des Sciences de l'Éducation, XXII(3), 467-502. 
Vézina, M., Cloutier, E., Stock, S. Lippel, K., Fortin, E., Delisle, A., ... Prud'homme, P. (2011). Enquête québécoise sur des conditions de travail, d'emploi et de santé et de sécurité du travail (EQCOTESST). Rapport, Études et recherches, R-691. Montréal, QC : IRSST.

Yvon, F. et Saussez, F. (2010). Analyser l'activité enseignante : Des outils méthodologiques et théoriques pour l'intervention et la formation. Québec, QC : Presses de l'Université Laval.

Yvon, F. et Garon, R. (2006). Une forme d'analyse du travail pour développer et connaître le travail enseignant : l'autoconfrontation croisée. Recherches Qualitatives, 26(1), 51-80.

\section{RÉSUMÉ}

Les risques pour la santé mentale en milieu scolaire au Québec sont nombreux et les salariés du secteur de l'enseignement seraient parmi les plus atteints par la détresse psychologique. Considérant que les interventions préventives individuelles sont insuffisantes et peinent à enrayer ce mal, nous présentons les fruits d'une recherche-intervention inspirée de la clinique de l'activité qui montre que la santé peut se construire par le pouvoir d'agir dans un contexte offrant la possibilité de générer de nouvelles normes de vie au travail. Les résultats feront état du développement du pouvoir d'agir individuel et collectif au sein d'un comité santé.

\section{MOTS CLÉS}

santé psychologique, personnels scolaires, recherche-intervention, pouvoir d'agir.

\section{ABSTRACT}

While Quebec school employees' mental health is at risk, interventions do not seem to be able to reduce the problem. Considering that preventive individual interventions are insufficient to reduce at the source the psychological health problems at work, we present an intervention-research inspired by the clinic of activity that shows that health can be produced by the power to act in a context providing the ability to generate new work life norms. The results show the individual and collective development of the power to act within a Health Committee.

\section{KEY WORDS}

psychological health, school employees, intervention-research, power to act. 